\title{
Improved Accuracy for Interferometric Radar Images Using Polarimetric Radar and Laser Altimetry Data
}

\author{
K. Clint Slatton ${ }^{1,2}$, Melba M. Crawford ${ }^{1,3}$, and Brian L. Evans ${ }^{2}$ \\ ${ }^{1}$ Center for Space Research, The University of Texas at Austin \\ ${ }^{2}$ Dept. of Electrical and Computer Engineering, The University of Texas at Austin \\ ${ }^{3}$ Dept. of Mechanical Engineering, The University of Texas at Austin \\ E-mail: slatton@ csr.utexas.edu, Ph: +1.512.471-5509, Fax: +1.512.471-3570
}

\begin{abstract}
The ability to measure land surface topography over large areas to assess natural hazard threats posed by seismic and flooding events is a critical, international need. Interferometric synthetic aperture radar (INSAR) has been used to map topography; however, accuracies are limited because observations are not measurements of true surface topography over vegetated areas. Instead, the measurements, which depend on the sensor and the vegetation, represent some height above the true surface. We develop a two-step correction for the INSAR imagery to account for penetration into the vegetation. The INSAR imagery is first adaptively filtered to reduce random measurement noise. We then combine the INSAR with polarimetric radar and laser altimetry data to account for the vegetation contribution to the topographic heights.
\end{abstract}

\section{Introduction}

There is a critical need to measure and monitor land surface topography over large areas to assess the threat and impact of natural hazards such as flooding. Imaging radars have been used extensively to map terrain because they can operate in the microwave portion of the electromagnetic spectrum, thereby enabling them to image during the day or night and under most weather conditions.

Both interferometric and stereo synthetic aperture radar (SAR) data can be used to determine topography over large areas, but interferometric SAR (INSAR) data provides the best option for making primary topographic measurements in low-relief areas due to its better vertical resolution. Although the capability of INSAR for mapping topography has been demonstrated, success has been primarily limited to areas where the surface is not obscured by significant vegetation [1]. For many applications, topographic features of interest occur in heavily vegetated regions, e.g. forests. Unfortunately, INSAR observations do not provide direct measurements of the true surface topography in vegetated areas, but instead yield a height $z_{s}$ that depends on the sensor characteristics, the surface elevation $z_{g}$, and the vegetation height $z_{v}$.

Vertical height accuracies of $2-5 \mathrm{~m}$ can be obtained in non-vegetated regions with airborne INSAR data processed to $10 \mathrm{~m} \times 10 \mathrm{~m}$ terrain patches (pixels), but the presence of vegetation can lead to errors in the computed surface topography of tens of meters in forested areas. A method is needed to distinguish surface elevations $z_{g}$ and vegetation heights $z_{v}$ from $z_{s}$. One approach is to develop a functional relationship to relate the INSAR observations to $z_{g}$ and $z_{v}$ using an electromagnetic scattering model [2]. Estimating the parameters $z_{g}$ and $z_{v}$ from the observations is then equivalent to inverting the model. An alternative approach is to combine INSAR with polarimetric SAR (POLSAR) and laser altimetry (LIDAR) data to derive corrections for $z_{s}$ to approximate $z_{v}$, which we explore in this paper. The key contribution of this paper is the development of a vegetation correction for INSAR imagery based on POLSAR and LIDAR data.

\section{Measurement types}

Imaging radar is a side-looking, active sensor, which means that it transmits and receives its own energy. Conventional SAR provides images of reflected power by binning the return echoes according to the time of arrival to determine the range position of targets. During synthetic aperture processing, this range gating affects the pixel location in the range dimension of the SAR image. It is not possible to determine actual distances to the targets with this type of imaging alone. Time of arrival and antenna altitude (determined independently) can be used to determine distance to the first pixel, but a position ambiguity remains. The subsequent pixels in the range dimension are simply mapped linearly according to the time of arrival onto what is termed the slant-range line.

It is, however, possible to accurately determine positions if two SAR images are combined interferometrically. An INSAR platform, such as an 
aircraft, operating in single-pass mode has two antennas that image the terrain simultaneously (see Figure 1). Quadrature demodulation and synthetic aperture processing of the received signals yield two complex-valued images $C_{1}\left[n_{1}, n_{2}\right]$ and $C_{2}\left[n_{1}, n_{2}\right]$, where $n_{1}$ and $n_{2}$ denote pixel coordinates. The interferometric cross correlation $C_{1}\left[n_{1}, n_{2}\right] C_{2}^{*}\left[n_{1}, n_{2}\right]$ is the primary INSAR measurement. The cross correlation phase is equal to the relative phase difference between the two images. After phase unwrapping, it is used to solve for the topographic heights $z_{s}$ and horizontal distances $y_{s}$ relative to the radar position through geometry [1].

Over barren surfaces, the phase difference used to determine $z_{S}$ will have a relatively small variance dictated by measurement noise. Over vegetated terrain, the INSAR signal can scatter from both the ground surface and vegetation constituents, e.g. tree trunks and branches. Therefore, the value of $z_{s}$ cannot be related to the physical topographic or vegetation heights from the phase data alone. In this work, LIDAR and POLSAR data are introduced to infer $z_{v}$ from $z_{s}$ imagery. The NASA/JPL Airborne SAR (AIRSAR) sensor provided polarimetric and interferometric data at L-band $(\lambda=24 \mathrm{~cm})$ and C-band $(\lambda=$ $5.7 \mathrm{~cm})$. The Optech, Inc. Airborne Laser Terrain Mapping (ALTM) system acquired laser altimetry data at $\lambda$ $=1047 \mathrm{~nm}$.

LIDAR is typically a down-looking, active sensor that illuminates the terrain with a dense pattern of laser pulses. Heights corresponding to each pulse are determined based on the round trip travel time (see Figure 2). For this study, the data was gridded into $10 \mathrm{~m} \times 10 \mathrm{~m}$ pixels to correspond to the SAR images, with several pulses corresponding to each pixel. The LIDAR data provides a mean height value $z_{L}$ for each pixel. Figures 3 and 4 contain $90 \times 90$ pixel co-registered images of INSAR and LIDAR data over a vegetated test site near the Texas coast. The LIDAR resolves the vegetation and topography better in both the horizontal and vertical dimensions. This is due both to the small footprint of the laser and lack of penetration of the canopy at the short wavelengths of the optical portion of the spectrum. In vegetated areas, LIDAR is primarily sensitive to the absolute height of the vegetation, but not to the vegetation structure or height of the surface below the vegetation. Surface heights are measured where gaps exist in the vegetation cover.

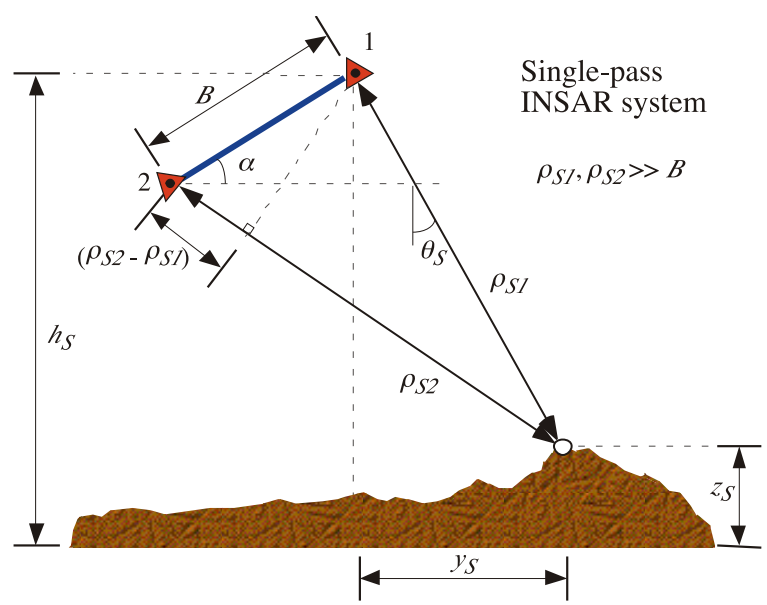

Figure 1. INSAR imaging geometry, where $h_{s}$ is the altitude of antenna $1, B$ is the baseline distance, $\alpha$ is the baseline angle, $\theta_{s}$ is the incidence angle, $\rho_{s 1}$ and $\rho_{s 2}$ are the path lengths from antennas 1 and 2, respectively, and $y_{s}$ and $z_{s}$ are the target coordinates. The azimuth direction is out of the plane, and the range direction is to the right.

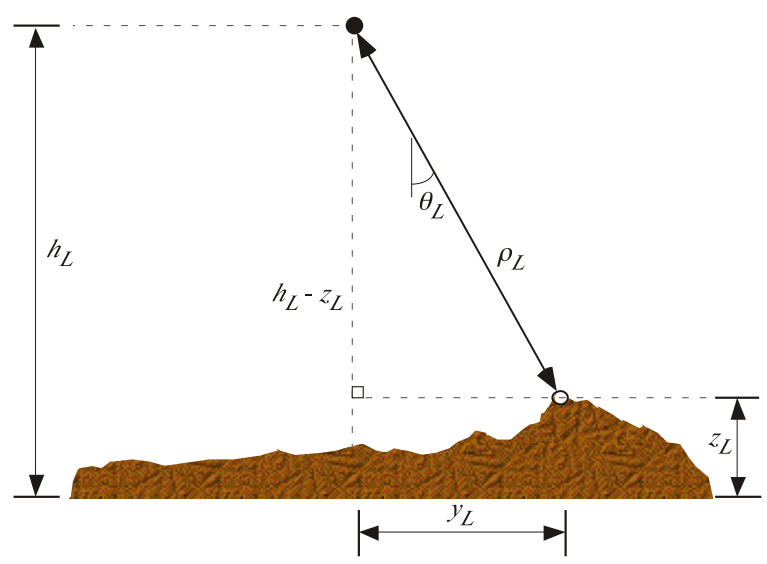

Figure 2. LIDAR imaging geometry, where $h_{L}$ is the altitude of sensor, $\theta_{L}$ is the incidence angle, $\rho_{L}$ is the path length from the sensor, and $y_{L}$ and $z_{L}$ are the target coordinates.

Typical vertical accuracy for single-pass airborne INSAR is $2-5 \mathrm{~m}$ over level, non-vegetated terrain, with 10 $\mathrm{km}$ imaging swaths. This random measurement error is due primarily to thermal noise and spatial decorrelation due to the non-identical viewing directions of the two antennas. LIDAR systems can yield $10 \mathrm{~cm}$ vertical accuracy, but imaging swaths are generally less than $0.5 \mathrm{~km}$. The INSAR height image in Figure 3 and the corresponding LIDAR height image in Figure 4 are displayed using the same linear histogram stretch so that equivalent pixel values correspond to equivalent heights. 
We do not use the full-resolution LIDAR data to correct the INSAR because complete LIDAR coverage is not typically available everywhere there is SAR coverage. Instead, we relate deviations between $z_{S}$ and $z_{L}$ to coregistered POLSAR images. POLSAR imagery at different wavelengths and polarizations can be used to indicate the presence of vegetation [3].

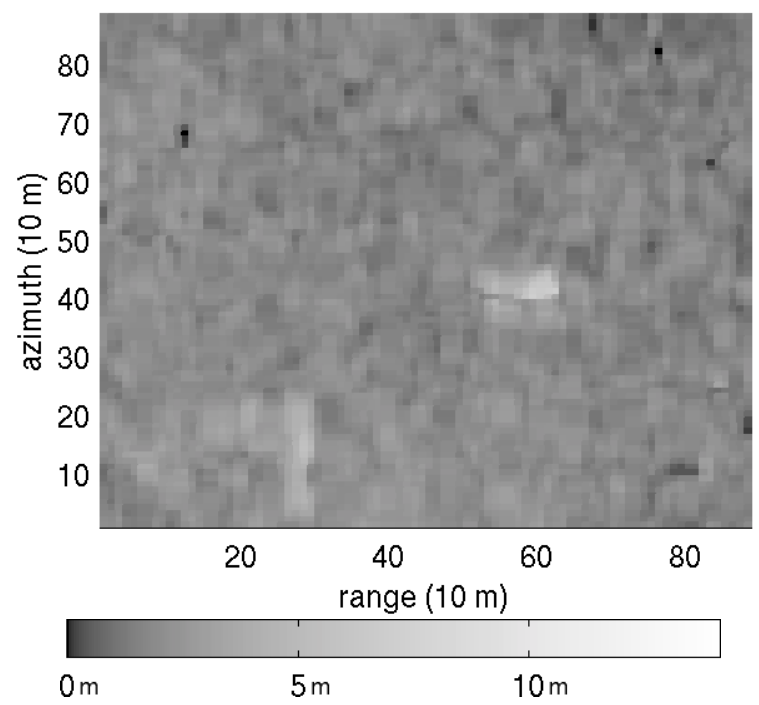

Figure 3. Image of $z_{s}$, acquired at C-band

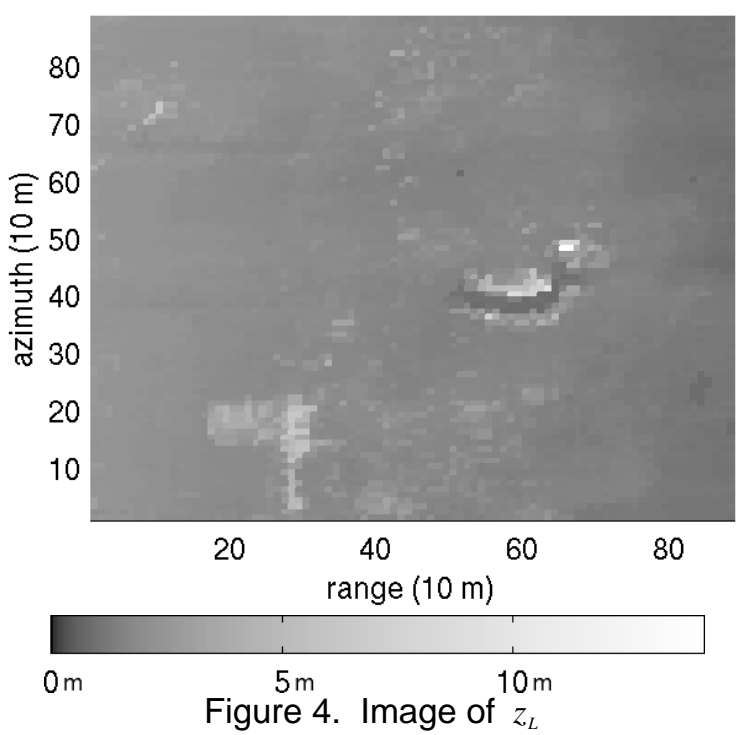

\section{Correcting INSAR for vegetation}

Recent attempts to account for vegetation based on regression and empirical modeling have achieved some success but were limited to repeat-pass INSAR data [4], [5]. Interferometric phase, correlation, and the single- polarization backscatter data used to derive the interferograms were classified into general land cover types using INSAR data from the first European Remote Sensing (ERS-1) satellite [4]. Results were affected by temporal decorrelation that occurs because of the changes in the scene between acquisitions. Because temporal decorrelation can mimic low correlation due to scattering from vegetation, it is then more difficult to determine heights. This paper is concerned with investigating the relationships among a variety of complementary data sources, including POLSAR and LIDAR, over vegetated terrain. This richer set of data makes it possible to "correct" $z_{S}$ images to obtain approximate $z_{v}$ images.

The SAR and LIDAR images were co-registered, and a $90 \times 90$ pixel subset over the vegetated test site was examined. Preliminary processing was required to remove a vertical offset and 2-D tilt from the INSAR $z_{S}$ image relative to the LIDAR. The LIDAR image was first downsampled by a factor of ten to represent the typical case of sparse coverage. Mosaicked LIDAR data does not exhibit global tilts because of the sensor's down-looking acquisition geometry and small imaging swaths.

\subsection{INSAR noise reduction}

INSAR data over barren terrain can exhibit height errors of the same order of magnitude as typical vegetation heights. It is therefore crucial to minimize this random sensor noise prior to determining vegetation heights. The INSAR measurement errors noted in Section 2 are aggregated into a single sensor-noise parameter for the $z_{S}$ image. An additive, zero-mean, white Gaussian process can be used to model the height errors that are not attributable to vegetation [5]. Although uniform distributions are often used to model phase noise, Gaussian distributions are appropriate for height errors, which are linearly proportional to the phase-difference noise [6], [7]. A Minimum Mean Squared Error (MMSE) filter was used to reduce the noise in the $z_{S}$ image.

Surface topography measurements are realizations of a two-dimensional, non-stationary random process, which can be characterized by the autocorrelation function [8]. When considering vertical relief of a few meters or more, the minimum separation distance between two nearly statistically independent points (correlation length) is often longer than the INSAR pixel spacing [9].

Patches over which the signal is considered locally wide sense stationary are filtered in the spatial domain using an adaptive FIR filter [10]. The variance of the noise was estimated from image pixels over level cut fields. The sample variances of the observed signal and of the noise were then used to compute the filter coefficients for a $3 \times 3$ window. The filtered INSAR image is denoted by $z_{S f}$. 


\subsection{Vegetation correction}

After the random noise was filtered, the POLSAR and LIDAR data were used to determine $z_{v}$ from $z_{s f}$. Because LIDAR heights correspond roughly to the tops of the vegetation, and INSAR penetrates into the vegetation, the remaining mean squared error (MSE) between the INSAR and LIDAR is attributed predominantly to the presence of vegetation. Pixels affected by vegetation were identified in the POLSAR imagery. Corrections to the heights were then determined using downsampled LIDAR data.

Differences between $z_{S f}$ and $z_{L}$ correlated with the Lband, multi-polarization POLSAR data. Results were class-dependent, so classification results obtained previously from C- and L-band data were employed to identify vegetated pixels [3]. The affected pixels were labeled as members of three terrain archetypes based on vegetation height: barren, medium height, and trees.

INSAR heights over vegetated areas were then incremented by class-dependent average deviations between the INSAR and LIDAR to offset penetration through the vegetation. The LIDAR pixels in each class were first filtered with a 3-tap median filter. This was done because the higher spatial resolution of the LIDAR caused trees and the gaps between trees to be included in the tree class. The median filter reduced the effect of the gaps on the height increment for the tree class.

The INSAR height image, corrected for vegetation $z_{v}$, is shown in Figure 5. The same histogram stretch was used in Figures 3-5. Not every gray level is realized by $z_{v}$ under this histogram stretch because the range of heights is determined from both LIDAR and INSAR data. A transect corresponding to column 23 of the $z_{v}$ image is shown in Figure 6 to illustrate the results more clearly. The height offsets are given in Table 1 and the error results are given in Table 2. The quantity $\Delta \mathrm{MSE}_{\text {Global }}$ refers to percent reduction in global MSE from unfiltered $z_{s}$. The vegetation correction improved $\Delta \mathrm{MSE}_{\mathrm{Global}}$ an additional $6 \%$ over MMSE filtering. The vegetation correction is not guaranteed to reduce the difference between $z_{S f}$ and $z_{L}$ for a particular pixel or group of pixels. It did, however, reduce the MSE for each class and for the entire image presented in Figures 3-5, as well as other locations in the test area.

It can be seen from Table 2 that there was more improvement over barren and tree classes than over the medium foliage class. The height offset for barren terrain is negative and the offset for trees is positive. The positive offset for the trees occurs because the penetration of the longer wavelength INSAR leads to an underestimating of the height where tall vegetation is present. The negative offset over barren areas is most likely due to the LIDAR and INSAR being acquired at different times of the year. The LIDAR data was acquired in November, when the grasslands in the test site had senesced. The grasslands were probably slightly denser in the spring of the preceding year, when the INSAR was acquired. The denser vegetation could have caused a small positive bias in the INSAR heights.

Figure 6 indicates that the correction for the tree class was too small. This is because the single class for trees in the original classification included both tall shrubs and trees with varying degrees of percent cover. This was a consequence of including C-band POLSAR, which can scatter strongly even from short vegetation, in the classification and the selection of training sites for the classifier. Consequently, some short vegetation was included in the determination of the height increment for the tree class. Reclassifying the POLSAR imagery using only L-band and training the classifier more specifically on trees should improve results.

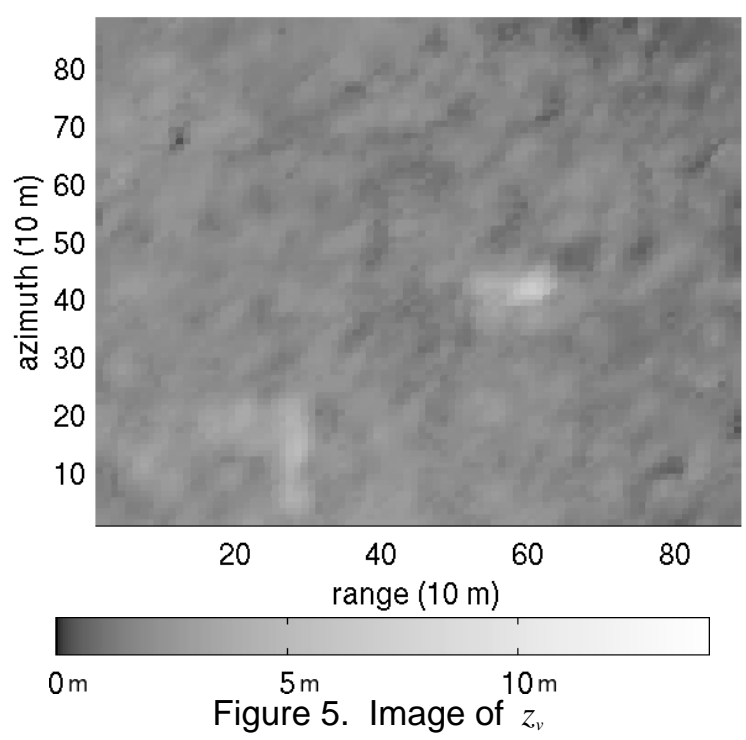




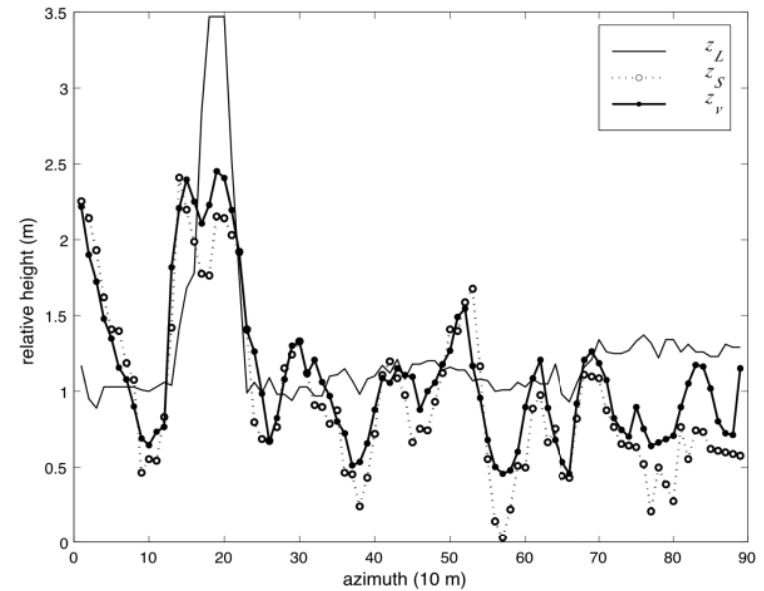

Figure 6. Transects from column 23 of the test images. The corrected INSAR heights $z_{v}$ generally approximate $z_{L}$ better than the uncorrected INSAR $z_{s}$ for all vegetation heights.

Table 1. Height offsets added to $z_{\text {sf }}$
\begin{tabular}{|l|r|}
\hline Barren & $-0.23 \mathrm{~m}$ \\
\hline Medium & $0.03 \mathrm{~m}$ \\
\hline Trees & $0.20 \mathrm{~m}$ \\
\hline
\end{tabular}

Table 2. MSE results relative to $z_{L}$ after noise reduction $z_{s f}$ and after noise reduction plus vegetation correction $z_{v}$. $\Delta \mathrm{MSE}_{\mathrm{Global}}$ is percent reduction in global MSE from unfiltered $z_{s}$.

\begin{tabular}{|l|l|l|l|}
\hline \multicolumn{2}{|c|}{$z_{S f}$} & \multicolumn{2}{c|}{$z_{v}$} \\
\hline \hline $\mathrm{MSE}_{\text {Barren }}$ & 0.52 & $\mathrm{MSE}_{\text {Barren }}$ & 0.48 \\
\hline $\mathrm{MSE}_{\text {Meduim }}$ & 0.25 & $\mathrm{MSE}_{\text {Meduim }}$ & 0.25 \\
\hline $\mathrm{MSE}_{\text {Trees }}$ & 0.40 & $\mathrm{MSE}_{\text {Trees }}$ & 0.35 \\
\hline $\mathrm{MSE}_{\text {Global }}$ & 0.36 & $\mathrm{MSE}_{\text {Global }}$ & 0.33 \\
\hline$\Delta \mathrm{MSE}_{\text {Global }}$ & $13 \%$ & $\Delta \mathrm{MSE}_{\text {Global }}$ & $19 \%$ \\
\hline
\end{tabular}

\section{Conclusions}

A two-step algorithm was developed to correct INSAR height images for the presence of vegetation where:

1. measurement noise is reduced through adaptive filtering,

2. class-dependent increments are added to the INSAR heights.

The vegetation classes are identified using POLSAR data, and the increments are determined using LIDAR data.

Initial results from the vegetation correction show promise. A $6 \%$ reduction in the global mean squared error relative to MMSE filtering alone was achieved for the location in Figures 3-5. A larger improvement was achieved for another location, but many other test images will be analyzed to investigate the robustness of the vegetation corrections in different environments. The POLSAR imagery will be reclassified using only L-band data and retrained on the trees to improve corrections for the tree class. Similar approaches will be examined that use POLSAR data directly rather than classified images. The corrections will likely degrade, but the need for classifying the POLSAR data will be eliminated.

\section{Acknowledgements}

This work was supported in part by the National Aeronautics and Space Administration, under the Topography and Surface Change Program (Grant NAG52954).

\section{References}

[1] F. Li and R. M. Goldstein, "Studies of multi-baseline spaceborne interferometric synthetic aperture radars," IEEE Trans. Geosci. Remote Sensing, vol. 28, no. 5, pp. 88-97, 1990.

[2] R. N. Treuhaft and P. R. Siqueira, "Vertical structure of vegetated land surfaces from interferometric and polarimetric radar," Radio Science, vol. 35, no. 1, pp. 141-177, 1999.

[3] M. M. Crawford, S. Kumar, M. R. Ricard, J. C. Gibeaut, and A. Neuenschwander, "Fusion of Airborne Polarimetric and Interferometric SAR For Classification of Coastal Environments," IEEE Trans. Geosci. Remote Sensing, vol. 37, no. 3, pp. 1306-1315, 1999.

[4] U. Wegmuller and C. Werner, "Retrieval of vegetation parameters with SAR interferometry," IEEE Trans. Geosci. Remote Sensing, vol. 35, no. 1, pp. 18-24, 1997.

[5] J. O. Hagberg, L. M. Ulander, and J. Askne, "Repeat-pass SAR interferometry over forested terrain," IEEE Trans. Geosci. Remote Sensing, vol. 33, no. 2, pp. 331-40, 1995.

[6] J. S. Lee, K. P. Papathanassiou, T. L. Ainsworth, M. R. Grunes, and A. Reigber, "A new technique for noise filtering of SAR interferogram phase images," Proc. IEEE Int. Geosci. Remote Sensing Symp., Singapore, pp. 1716-18, 1997.

[7] N. P. Nikoluas and E. H. Meier, "First results with airborne single-pass DO-SAR interferometer," IEEE Trans. Geosci. Remote Sensing, vol. 33, no. 5, pp. 1230-7, 1995.

[8] J. J. van Zyl, C. F. Burnette, and T. Farr, "Inference of surface power spectra from inversion of multifrequency polarimetric radar data," Geophys. Res. Letters, vol. 18, no. 9, pp. 1787-90, 1991.

[9] P. C. Kyriakidis, A. M. Shortridge, and M. F. Goodchild "Geostatistics for conflation and accuracy assessment of digital elevation models," Int. J. Geographical Information Science, vol. 13, no. 7, pp. 677-707, 1999.

[10] J. S. Lim, Two-Dimensional Signal and Image Processing, Englewood Cliffs, NJ: Prentice Hall, 1990. 